\title{
PERFIL BIOQUÍMICO SANGUÍNEO HEPÁTICO DE VENADOS COLA BLANCA (ODOCOILEUS VIRGINIANUS) CRIADOS EN CAUTIVERIO
}

\author{
Liver Biochemistry Profile in White-Tailed Deer (Odocoileus virginianus) \\ REARED IN CAPTIVITY
}

\author{
David Alhuay A. ${ }^{1}$, Olga Li E. ${ }^{1,2}$, Arnaldo Alvarado S. ${ }^{1}$, Néstor Falcón P. ${ }^{3,4}$, \\ Patricia Ríos M. ${ }^{5}$, Gianmarco Rojas M. ${ }^{6}$
}

\section{RESUMEN}

El presente estudio se llevó a cabo con 23 venados cola blanca (Odocoileus virginianus), de ambos sexos, y criados en cautiverio en la provincia de Lima, con el objetivo de determinar el perfil bioquímico sanguíneo hepático a través de los valores séricos de Bilirrubina Total y Directa, Proteínas Totales, Albúmina, Alanino Amino Transferasa (ALT), Aspartato Amino Transferasa (AST), Fosfatasa Alcalina (FA), y Gamma Glutamil Transferasa (GGT). Los animales (7 machos y 16 hembras), pertenecientes a tres zoológicos o zoocriaderos, fueron anestesiados con clorhidrato de ketamina o con la combinación de clorhidrato de ketamina y clorhidrato de xilacina. Se extrajo $7 \mathrm{ml} \mathrm{de}$ sangre por punción de la vena safena en tubos estériles sin anticoagulante, y los sueros fueron analizados con kits comerciales. Los valores encontrados (media $\pm \mathrm{DE}$ ) fueron para Bilirrubina Total: $0.6 \pm 0.3 \mathrm{mg} / \mathrm{dl}$; Bilirrubina Directa: $0.08 \pm 0.06 \mathrm{mg} / \mathrm{dl}$; Proteínas Totales: $6.6 \pm 0.7 \mathrm{~g} / \mathrm{dl}$; Albúmina: $3.6 \pm 0.5 \mathrm{~g} / \mathrm{dl}$; ALT: $26.0 \pm 9.7 \mathrm{UI} / \mathrm{l}$; AST: $87.6 \pm 22.9 \mathrm{UI} / \mathrm{l}$; FA: $73.9 \pm 33.8 \mathrm{UI} / \mathrm{l}$; y GGT: $42.5 \pm 12.6 \mathrm{UI} / \mathrm{l}$. No hubo diferencia estadística entre sexos con excepción de BT y albúmina $(\mathrm{p}<0.05)$.

Palabras clave: Odocoileus virginianus, venado cola blanca, bioquímica hepática, valores referenciales

\section{Abstract}

This study was carried out with 23 adult white-tailed deer (Odocoileus virginianus) of both sexes, kept in captivity in the province of Lima to establish reference liver biochemistry profile values (Total and direct bilirubin, total protein, albumin, alanine amino transferase (ALT), aspartate amino transferase (AST), alkaline phosphatase (AF), and gamma glutamyl transferase (GGT). The animals ( 7 males and 16 females) were from

\footnotetext{
${ }^{1}$ Laboratorio de Patología Clínica y Biología Molecular, ${ }^{3}$ Laboratorio de Medicina Veterinaria Preventiva, Facultad de Medicina Veterinaria, Universidad Nacional Mayor de San Marcos, Lima

${ }^{2}$ E-mail: olgalie@hotmail.com

${ }^{4}$ Dirección actual: Facultad de Veterinaria y Zootecnia, Universidad Peruana Cayetano Heredia, Lima

${ }^{5}$ Parque Ecológico Santa Rosa de Lima y Zoocriadero del Colegio de la Inmaculada, Lima

${ }^{6}$ Zoológico Huachipa, Lima
} 
three local zoos and were anesthesyzed with ketamine hydrochloride or with a combination of ketamine hydrochloride and xylazine hydrochloride. Blood samples (7 $\mathrm{ml}$ ) were collected from the saphenous vein in sterile tubes and serum was analyzed using commercial kits. The values (mean $\pm \mathrm{SD}$ ) were Total Bilirubin: $0.6 \pm 0.3 \mathrm{mg} / \mathrm{dl}$; Direct Bilirubin: $0.08 \pm 0.06 \mathrm{mg} / \mathrm{dl}$; Total Protein: $6.6 \pm 0.7 \mathrm{~g} / \mathrm{dl}$; Albumin: $3.6 \pm 0.5 \mathrm{~g} / \mathrm{dl}$; ALT: $26.0 \pm 9.7 \mathrm{UI} / \mathrm{l}$; AST: $87.6 \pm 22.9 \mathrm{UI} / \mathrm{l}$; AF: $73.9 \pm 33.8 \mathrm{UI} / \mathrm{l}$, and GGT: $42.5 \pm 12.6 \mathrm{UI} / \mathrm{l}$. There were no statistical differences due to sex except for total bilirubin and albumin $(\mathrm{p}<0.05)$.

Key words: Odocoileus virginianus, white-tailed deer, liver biochemistry, reference values

\section{INTRODUCCIÓN}

La fauna del Perú es muy variada, y de gran importancia para el país, tanto en lo económico, social como científico (Brack y Mendiola, 2000). Dentro de las especies nativas, el venado cola blanca (Odocoileus virginianus) es muy apreciado como animal de caza por deporte, subsistencia o comercio de la carne, piel y astas, así como por su valor estético donde es usado como atractivo turístico en áreas protegidas o en zoológicos. Su fácil adaptabilidad a la vida en cautiverio, permite que no existan problemas en su mantenimiento ni en la reproducción; aunque en su manejo se debe tener especial cuidado ya que son susceptibles a sufrir problemas de estrés por su comportamiento nervioso (Guzmán, 2005). Además, diversas enfermedades y parásitos que afectan a esta especie tienen un efecto deletéreo potencial sobre humanos y animales domésticos. Entre estos se incluye el ántrax, fiebre aftosa, tuberculosis y algunos arbovirus (Fargione y Curtis, 1998; Palmer et al., 2002).

Desde el punto de vista de la salud pública, se tiene que tomar en consideración que el venado cola blanca es hospedero para las garrapatas que llevan las bacterias responsables de la enfermedad de Lyme Dewey y Animal Diversity Web Staff, 2003). Además, estudios experimentales y de campo lo han implicado como reservorio para Ehrlichia chaffensis, el agente causal de la ehrlichiosis monocítica humana (Lockhart et al., 1997; Dummler, 1999).
El manejo o control de las poblaciones de mamíferos silvestres se basa en monitoreos continuos de su estado demográfico, fisiológico y genético. Los valores de bioquímica sérica así como los de hematología pueden proporcionar, cuando son correctamente interpretados, un panorama preciso de las condiciones de un animal al momento de su muestreo (i.e., estado nutricional, estado de enfermedad, y nivel de estrés debido a la captura o manejo); sin embargo, para usar esta información se requiere compararla con valores referenciales o normales (White y Cook, 1974; Crooks et al., 2003; Pérez et al., 2003). Por lo tanto, el objetivo del presente estudio fue establecer el perfil bioquímico sanguíneo hepático en el venado cola blanca criado en cautiverio.

\section{Materiales y Métodos}

Se tomaron muestras sanguíneas de 23 venados cola blanca (Odocoileus virginianus), mayores de un año (7 machos y 16 hembras); seis de los cuales procedían del Zoológico Huachipa, nueve del Parque Ecológico Santa Rosa de Lima y ocho del Zoocriadero de la Inmaculada. Se hizo una evaluación clínica de los animales, incluyendo observaciones de comportamiento, apetito, consistencia y color de las heces, ausencia o presencia de secreciones nasales, y constantes fisiológicas para asegurar que estuvieran libres de enfermedad. 
Para la toma de las muestras en horario matutino, los animales fueron mantenidos en ayuno por 12 horas. Fueron sedados utilizando una cerbatana con dardos que contenían clorhidrato de ketamina, a razón de $10 \mathrm{mg} / \mathrm{kg}$ (Zoológico Huachipa) o una combinación de clorhidrato de ketamina $(4 \mathrm{mg} / \mathrm{kg})$ y clorhidrato de xilacina $(1 \mathrm{mg} / \mathrm{kg})$ en el Parque Ecológico Santa Rosa de Lima y Zoocriadero del Colegio de la Inmaculada. Se recolectó 7 $\mathrm{ml}$ de sangre de la vena safena en tubos estériles sin anticoagulante. Las muestras se llevaron en una caja térmica al Laboratorio de Patología Clínica de la Facultad de Medicina Veterinaria de la Universidad Nacional Mayor de San Marcos, Lima. Se centrifugaron a $3000 \mathrm{rpm}$ por 10 minutos y el suero resultante se guardó en congelación hasta su análisis.

Se realizaron pruebas de bioquímica hepática, utilizando kits comerciales (Wiener Lab). Se determinó bilirrubina total, bilirrubina directa, proteínas totales, albúmina, y las enzimas Alanino Amino Transferasa (ALT), Aspartato Amino Transferasa (AST), Fosfatasa Alcalina (FA) y Gamma Glutamil Transferasa (GGT).

Los resultados obtenidos fueron analizados mediante estadísticas descriptivas, empleando la media aritmética como medida de tendencia central y la desviación estándar, y el rango como medida de dispersión. Asimismo, se utilizó la prueba estadística "t" de Student para determinar diferencia estadística entre sexos; el análisis de varianza para determinar diferencia por efecto de la procedencia (zoológico o zoocriadero), y la prueba de Tuckey para determinar diferencias entre estratos.

\section{Resultados y Discusión}

Los promedios de bilirrubina total en machos fue de $0.8 \mathrm{mg} / \mathrm{dl}$ y en hembras de 0.5 $\mathrm{mg} / \mathrm{dl}(\mathrm{p}<0.05)$ y de bilirrubina directa fue de $0.08 \mathrm{mg} / \mathrm{dl}$, sin diferencia estadística entre sexos. Tampoco se encontró diferencia esta- dística en estos dos parámetros con relación al lugar de procedencia (Cuadros 1 y 2).

Los promedios hallados para proteína total y albúmina fueron de 6.6 y $3.6 \mathrm{~g} / \mathrm{dl}$, respectivamente (Cuadro 1), encontrándose diferencia significativa por efecto del sexo en los promedios de albúmina ( 3.3 y $3.8 \mathrm{~g} / \mathrm{dl}$ en machos y hembras, respectivamente, Cuadro 2).

Los promedios de ALT, AST, FA y GGT fueron 26.0, 87.6, 73.9 y 42.5 UI/L, respectivamente (Cuadro 1), sin diferencia estadística entre sexos ni lugar de procedencia. La excepción fue la enzima GGT, que se encontró un valor más bajo en el zoológico Huachipa (29.2 UI/L) con respecto al Parque Ecológico Santa Rosa de Lima (43 UI/ L) y al Zoocriadero de la Inmaculada (51.9 $\mathrm{UI} / \mathrm{L})(\mathrm{p}<0.05)$.

No se han realizado estudios de bioquímica sanguínea en venados cola blanca en el país, y en consecuencia, la discusión comparativa se ha hecho con valores encontrados en esta especie en los EEUU (White y Cook, 1974; Chao et al., 1984; Klinger et al., 1986); así como con datos del ISIS (1999), provenientes de exámenes anuales de laboratorio en animales aparentemente sanos, procedentes de diversos zoológicos de Europa y EEUU.

Los valores de bilirrubina total y directa del presente estudio fueron en promedio mucho más bajos que los valores reportados por el ISIS (1999) [BT: $1.4 \pm 0.8 \mathrm{mg} / \mathrm{dl}(0.2-$ 3.6); BD: $0.2 \pm 0.1 \mathrm{mg} / \mathrm{dl}(0.1-0.3)]$. Los valores de bilirrubina pueden ser afectados por la localidad geográfica, las condiciones climáticas, e incluso por las técnicas empleadas para su medición o el laboratorio que las realizó. Además, el efecto de algunos antibióticos (sulfonamidas y cefalosporinas), AINES (acetaminofén y fenilbutazona), así como la lipemia y la hemólisis en las muestras pueden incrementar los valores de BT en el suero, mientras que los valores disminuyen en animales tratados con ácido 
Cuadro 1. Valores de bioquímica hepática en el venado cola blanca (Odocoileus virginianus) criado en cautiverio en la zona de Lima

\begin{tabular}{lcccc}
\hline Variable & Número & Promedio \pm d.e. & $\begin{array}{c}\text { Valor } \\
\text { Mínimo }\end{array}$ & $\begin{array}{c}\text { Valor } \\
\text { Máximo }\end{array}$ \\
\hline Bilirrubina total (mg/dl) & 23 & $0.6 \pm 0.3$ & 0.3 & 1.1 \\
Bilirrubina directa (mg/dl) & 23 & $0.08 \pm 0.06$ & 0.05 & 0.26 \\
Proteínas totales (g/dl) & 23 & $6.6 \pm 0.7$ & 5.5 & 7.8 \\
Albúmina (g/dl) & 23 & $3.6 \pm 0.5$ & 2.8 & 4.6 \\
ALT (UI/l) & 23 & $26.0 \pm 9.7$ & 12.0 & 54.0 \\
AST (UI/l) & 23 & $87.6 \pm 22.9$ & 46.0 & 150.0 \\
Fosfatasa alcalina (UI/l) & 23 & $73.9 \pm 33.8$ & 13.0 & 136.0 \\
GGT (UI/l) & 23 & $42.5 \pm 12.6$ & 11.0 & 61.0 \\
\hline
\end{tabular}

Cuadro 2. Valores de bioquímica hepática en el venado cola blanca (Odocoileus virginianus) criado en cautiverio en la zona de Lima, de acuerdo al sexo (machos $=7$; hembras $=16$ )

\begin{tabular}{lcc}
\hline Variable & $\begin{array}{c}\text { Machos } \\
\text { Promedio } \pm \text { d.e. }\end{array}$ & $\begin{array}{c}\text { Hembras } \\
\text { Promedio } \pm \text { d.e. }\end{array}$ \\
\hline Bilirrubina total $(\mathrm{mg} / \mathrm{dl})$ & $0.8 \pm 0.3$ & $0.5 \pm 0.2$ \\
Bilirrubina directa $(\mathrm{mg} / \mathrm{dl})$ & $0.09 \pm 0.08$ & $0.08 \pm 0.03$ \\
Proteínas totales $(\mathrm{g} / \mathrm{dl})$ & $6.3 \pm 0.8$ & $6.8 \pm 0.7$ \\
Albúmina $(\mathrm{g} / \mathrm{dl})$ & $3.3 \pm 0.3$ & $3.8 \pm 0.5$ \\
ALT $(\mathrm{UI} / \mathrm{l})$ & $30.6 \pm 13.1$ & $23.9 \pm 7.4$ \\
AST $(\mathrm{UI} / \mathrm{l})$ & $89.0 \pm 31.1$ & $86.9 \pm 19.6$ \\
Fosfatasa alcalina $(\mathrm{UI} / \mathrm{l})$ & $74.1 \pm 35.4$ & $73.8 \pm 34.2$ \\
GGT $(\mathrm{UI} / \mathrm{l})$ & $48.4 \pm 9.0$ & $39.9 \pm 13.3$ \\
\hline
\end{tabular}

ascórbico (Meyer y Harvey, 1998). También se ha reportado un aumento considerable de la BT sérica en caballos sometidos a ayuno de 24 horas (Benjamín, 1991).

Los valores de proteína total fueron similares a los reportados por ISIS (1999), aunque ligeramente superiores a los promedios de 6.4 y $6.2 \mathrm{~g} / \mathrm{dl}$ encontrados por White y Cook (1974) y Klinger et al. (1986), respectivamente. Sin embargo, se estima que los valores normales de casi todos los animales varían entre 5 y $8 \mathrm{~g} / \mathrm{dl}$ (Benjamín, 1991). No obstante, hay que considerar que una hipoproteinemia relativa puede ser consecuencia de un aumento de la cantidad de agua en la sangre; y una hiperproteinemia relativa suele observarse en casos de deshidratación, siendo ambos cambios fisiológicamente normales (IICA, 1989). Los valores de albúmina fueron ligeramente superiores a los encontrados en otros estudios (Klinger et al., 1986; ISIS, 1999) y similares a otros (White y Cook, 1974). Indudablemente, el tipo de die- 
ta influye en los valores de albúmina (Meyer y Harvey, 1998), así como una disminución de la ingesta de líquidos (Bush, 1982).

Los valores de ALT fueron menores a los reportados por el ISIS (1999) [46 \pm 23 $\mathrm{UI} / \mathrm{l}$ (14-101)], al igual que los valores de AST [135 \pm 82 UI/l (61-424), ISIS (1999); 254 UI/ 1, Klinger et al., 1986)]. Si bien se observan diferencias, la variabilidad individual en todos los estudios, incluyendo el presente, son bastante grandes. Además, las diferencias pueden atribuirse a las condiciones climáticas y geográficas, variaciones individuales de cada animal; así como al manejo y procesamiento de las muestras (Meyer y Harvey, 1998). El nivel de estas enzimas puede disminuir, además, por la deficiencia de piridoxina y al uso de ciertas drogas (fenotiacina y cefazolina), mientras que su incremento puede estar relacionado al efecto de corticosteroides, antibióticos (gentamicina, eritromicina y sulfonamidas) y AINES (ibuprofeno y fenilbutazona) (Benjamín, 1991). Por otro lado, valores bajos son indicadores de integridad hepática (Doxey, 1987).

Los valores de FA siguieron un perfil similar a las anteriores enzimas en relación con los valores reportados en la literatura (Chao et al., 1984; Klinger et al., 1986; ISIS, 1999). Es importante tener en consideración que los valores de FA se incrementan debido al empleo de corticosteroides y antibióticos como la eritromicina y sulfonamidas (Benjamín, 1991); aunque estas sustancias no han sido empleadas en los animales muestreados. Por último, los valores de GGT fueron menores a los reportados por el ISIS (1999).

La mayor concentración de BT sérica obtenida en machos podría haber estado influenciada por un mayor tiempo de ayuno, sobre todo en aquellos venados provenientes del Zoocriadero de la Inmaculada, donde hubo mayor cantidad machos. Meyer y Harvey (1998) mencionan que caballos sometidos a ayuno durante más de 24 horas experimentan un aumento considerable en la concentración sérica de bilirrubina no conjugada, incrementando así el valor de la BT sérica. Es posible que los ácidos grasos acumulados durante el ayuno interfieran la captación de bilirrubina no conjugada con el ácido glucorónico.

El presente estudio permitió establecer un perfil bioquímico sanguíneo para evaluar la función hepática en el venado cola blanca (Odocoileus virginianus) mantenido en cautiverio. Asimismo, no se encontró diferencia significativa entre machos y hembras en cuanto a los valores de bioquímica sanguínea, excepto para Bilirrubina Total y Albúmina.

\section{Literatura Citada}

1. Benjamín M. 1991. Manual de patología clínica veterinaria. México: Ed. Limusa. 423 p.

2. Brack A, Mendiola C. 2000. Ecología del Perú. Programa de las Naciones Unidas para el desarrollo. Lima, Perú.

3. Bush M. 1982. Manual del laboratorio veterinario de análisis clínico. Zaragoza, España: Acribia. 467 p.

4. Crooks K, Garcelon D, Scott C, Wilcox C, Timm S, Van Vuren D. 2003. Hematology and serum chemistry of the Island Spotted Skunk on Santa Cruz Island. J Wildlife Dis 39: 460-466.

5. Chao C, Brown R, Deftos L. 1984. Effects of xylazine immobilization on biochemical and endocrine values in whitetailed deer. J Wildlife Dis 20: 328-332.

6. Dewey T, Animal Diversity Web Staff. 2003. «Odocoileus virginianus». The Animal Diversity Web. University of Michigan Museum of Zoology. [Internet]. Disponible en: http://animaldiversity.ummz.umich.edu/site/accounts/ information/Odocoileus_virginianus.html

7. Doxey DL. 1987. Patología clínica y procedimientos de diagnóstico en veterinaria. $2^{\mathrm{a}}$ ed. México: Ed. Manual Moderno. $371 \mathrm{p}$.

8. Dummler J. 1999. The ehrlichioses: an overview. Infect Dis Rev 1(2): 110-112. 
9. Fargione M, Curtis P. 1998. Animal damage control: white-tailed deer. Department of Natural Resources, Cornell University. Ithaca, New York, USA. [Internet]. Disponible en: http:// www.hort.uconn.edu/IPM/general/htms/ deer.htm

10. Guzmán A. 2005. Análisis de las experiencias colombianas de manejo ex situ de venado cola blanca (Odocoileus virginianus) como aporte a su conservación. Tesis de Biología. Bogotá: Facultad de Biología, Univ. Nacional de Colombia. 280 p.

11. [IICA] Instituto Interamericano de Cooperación para la Agricultura. 1989. Patología clínica veterinaria. Seminario-Taller sobre Patología Clínica Veterinaria. Asunción, Paraguay.

12. [ISIS] International Species Information System. 1999. Reference ranges for physiological data values. Clinical pathology records report-ISIS/ In house reference values mammals. [Internet]. Disponible en: http:// www.worldzoo.org

13. Klinger $S$, Robel R, Brown B, Brent B. 1986. Blood characteristics of white- tailed deer from northeastern Kansas. J Wildlife Dis 22: 385-388.

14. Lockhart J, Davidson W, Stallknecht D, Dawson J, Howerth E. 1997. Isolation of Ehrlichia chaffensis from wild white tailed deer (Odocoileus virginianus) confirms their role as natural reservoir hosts. J Clin Microbiol 35: 1681-1686.

15. Meyer D, Harvey J. 1998. Veterinary laboratory medicine. Interpretation and diagnosis. $2^{\text {nd }}$ ed. Philadelphia, USA: WB Saunders. 373 p.

16. Palmer M, Gosch G, Lyon R, Waters $W$, Whipple D. 2002. Apoptosis in lymph node granulomas from white-tailed deer (Odocoileus virginianus) experimentally infected with Mycobacterium bovis. J Comp Pathol 127: 7-13.

17. Pérez J, González F, Granados J, Pérez C, Fandos F, Soriguer R, Serrano E. 2003. Hematological and biochemical reference intervals for Spanish ibex. J Wildlife Dis 39: 209-215.

18. White M, Cook R. 1974. Blood characteristics of free-ranging whitetailed deer in Southern Texas. J Wildlife Dis 10: 18-24. 